\title{
Vital and Clinical Signs Gathered Within the First Minutes After a Motorcycle Accident on a Racetrack: an Observational Study
}

\author{
Karin Hugelius ${ }^{1 *}$ (D), Jerry Lidberg ${ }^{2,3}$, Linda Ekh ${ }^{3,4}$ and Per Örtenwall ${ }^{5}$
}

\begin{abstract}
Background: Little is known about vital signs during the very first minutes after an accident. This study aimed to describe the vital signs of motorcycle riders shortly after racetrack crashes and examine the clinical value of these data for the prehospital clinical assessments.

Methods: A retrospective observational cohort based on data from medical records on 104 motorcycle accidents at a racetrack in Sweden, covering the season of 2019 (May 01 until September 17), was conducted. Both race and practice runs were included. In addition, data from the Swedish Trauma Registry were used for patients referred to the hospital. Kruskal-Wallis test and linear regression were calculated in addition to descriptive statistics.

Results: In all, 30 riders (29\%) were considered injured. Sixteen riders (15\%) were referred to the hospital, and of these, five patients (5\% of all riders) had suffered serious injuries. Aside from a decreased level of consciousness, no single vital sign or kinematic component observed within the early minutes after a crash was a strong clinical indicator of the occurrence of injuries. However, weak links were found between highsider or collision crashes and the occurrence of injuries.

Conclusion: Except for a decreased level of consciousness, this study indicates that the clinical value of early measured vital signs might be limited for the pre-hospital clinical assessment in the motorsport environment. Also, an adjustment of general trauma triage protocols might be considered for settings such as racetracks. Using the context with medical professionals at the victim's side within a few minutes after an accident, that is common in motorsport, offers unique possibilities to increase our understanding of clinical signs and trauma in the early state after an accident.
\end{abstract}

Keywords: Trauma, Motorcycle accidents, Vital signs, Prehospital care, Ambulance, Trauma triage

\section{Key Points}

- Except for a decreased level of consciousness, this study indicates that the clinical value of early measured vital signs to determine the severity of injuries after road racing crashes on racetracks might be limited.

\footnotetext{
* Correspondence: karin.hugelius@oru.se

${ }^{1}$ Faculty of Medicine and Health, Örebro University, 70182 Örebro, SE, Sweden

Full list of author information is available at the end of the article
}

- Type of crash seems to be more significant than speed as indicators for severe injuries on motorcycle crashes at racetracks.

- Study settings with medical professionals at the victim's side within a few minutes after an accident offers unique possibilities to increase our understanding of clinical signs and trauma.

\section{Background}

In 2016, 1.35 million persons globally were killed in road traffic deaths. Of these, about $28 \%$ were motorcycle riders [1]. In Sweden, 47 motorcyclists were killed and

\section{Springer Open}

(c) The Author(s). 2021 Open Access This article is licensed under a Creative Commons Attribution 4.0 International License, which permits use, sharing, adaptation, distribution and reproduction in any medium or format, as long as you give appropriate credit to the original author(s) and the source, provide a link to the Creative Commons licence, and indicate if changes were made. The images or other third party material in this article are included in the article's Creative Commons licence, unless indicated otherwise in a credit line to the material. If material is not included in the article's Creative Commons licence and your intended use is not permitted by statutory regulation or exceeds the permitted use, you will need to obtain permission directly from the copyright holder. To view a copy of this licence, visit http://creativecommons.org/licenses/by/4.0/. 
over 200 were injured during 2018 [2]. Road racing is a motorsport where motorcycles race on paved road surfaces, most often on a racetrack. However, little is known on the prevalence and outcome of accidents from road racing track accidents. One study on crashed in road racing grand prix indicates that $12-14 \%$ of all riders suffered a crash during racing [3]. In accordance with road racing regulations, medical teams are often present at racetracks to ensure an immediate emergency response in case of a crash [4]. In such situations, the first professional medical assessment can be made within a few minutes; this is opposed to motorcycle crashes on public roads, where the first public ambulance might arrive significantly later [5].

Prehospital assessment of trauma patients is a crucial part of the trauma care chain [6]. Most prehospital assessment protocols use a combination of vital signs, detection of certain anatomic injuries, injury mechanism and (sometimes) age [5]. However, little is known about vital and clinical signs in the very early stage after an accident, due to lack of studies and data. Kinetic energy is related to the square of the velocity of a moving object. On racing tracks, speeds may be in the range of $200 \mathrm{~km} /$ $\mathrm{h}$, with riders having corresponding extreme energy levels. To address this, protection measures, including safety zones and air fences (inflatable air barriers that protect bikers from hitting a solid fence), are designed to gradually reduce speed and thus energy. Moreover, requirements for wearing personal protective gear, such as helmets, have been introduced to minimize serious injuries $[7,8]$. These aspects might affect the clinical assessment of road racing riders. By describing and analysing vital and clinical signs within a few minutes of an accident, a greater understanding of their value for the prehospital clinical assessment in the early state after an accident can be obtained.

This study aimed to describe the vital and clinical signs of motorcycle riders shortly after racetrack crashes and examine the clinical value of these data for prehospital clinical assessments.

\section{Methods}

A retrospective observational cohort study was conducted by analysing medical records from 104 motorcycle crashes on a Swedish racetrack during the road racing season of 2019.

\section{Study Setting}

The racetrack used in this study was a permanent racetrack in Sweden. The medical teams consisted of nurses and physicians with experience in prehospital trauma care. In order to drive a motorcycle at the racing track, personal protective gear, such as a helmet and back protectors, was required.

\section{Participants}

Data from all motorcycle crashes on the racetrack during the season of 2019 (May 01 until September 17)when a medical team was present, and therefore, a medical record existed-were included. Crashes from races (national championships and regional/ local competitions) as well as practice runs were included. No private or commercial testing activities occurred on the actual racetrack during the season.

\section{Data Collection Procedure}

Data were collected retrospectively from paper medical records taken at the racetrack. Information on vital signs, clinical injury signs, type of crash and speed at crash was collected from the paper records and imported to the statistical programme SPSS (IBM Corp., released 2017. IBM SPSS Statistics for Windows, Version 25.0. Armonk, NY: IBM Corp). Three types of crashes [7] were studied: lowsider crashes, or crashes resulting from a loss of traction, where the motorcycle went down on the side closer to the ground; highsider crashes, involving sudden and violent long bike axis rotations, where the bike was oversteered into a turnover; and collisions with other bikers. If the riders were unable to specify actual speeds for their crashes, an estimation based on the specific location around the racetrack was used. For this purpose, time-tracking systems and helmet cameras providing speed measurements from ten professional and semi-professional motorcycle riders were used to determine the mean speed at all locations around the racetrack.

All vital signs were measured by members of the medical team. Clinical signs of injury were defined as documented pain or observed obvious injuries, such as paradoxical breathing, extremity deformities or external bleeding. For patients referred to an emergency hospital, information on their condition (measured with injury severity score (ISS) [9] and new injury severity score (NISS) [9]), documented type of injuries and status at discharge from hospital was collected from the Swedish Trauma Registry (SweTrau) and integrated into the SPSS dataset.

In order to ensure that all serious accidents at the racetrack during the observed season were included, the Nordic Motorsport Council and the insurance company that covers licensed racing motorcyclists were contacted and asked if they had any knowledge of reported injuries or fatalities of motorcycle drivers due to crashes at that specific racetrack aside from those already included. In addition, public ambulance alarm records for the racetrack were also scanned for accidents not included in the current data. 


\section{Analysis}

For descriptive analysis, percent proportions and, when applicable, means, ranges, standard deviations (SD) and a $95 \%$ confidence interval $(\mathrm{CI})$ were used to describe participant demographics, injuries and other information. Due to a small study sample and outliers, the Kruskal-Wallis test was used to analyse any differences between vital sign means among uninjured, injured and severely injured riders. Bonferroni post hoc analysis was used to determine significant differences between groups. Multiple regression analyses were conducted to calculate the association of speed at crash, type of crash, age of the rider, occurrence of clinical signs of injury and abnormal first vital signs (GCS, RR, Sp02, HR and $\mathrm{SBP}$ ) measured within the first $10 \mathrm{~min}$ from the crash $(n=62)$ with the occurrence of injuries (dependent variable). The exact analysis used and outcome variables are presented in each table or in the results. Injuries were defined as any signs of injury documented in the medical records or recorded, based on ISS, in the SweTrau. Mild injuries were defined as those with an ISS of 1-14, and severe injuries were defined as those with an ISS > 15 [7]. A confidence interval of $95 \%$ and a statistical significance of $p \leq 0.005$ were used with regard to the limited observations included [10]. No post hoc test was conducted, but the statistical analysis was verified by an external statistician.

Ethical approval was obtained from the Swedish Ethical Review Authority (dnr 2019-05803).

\section{Results}

Data from 104 motorcycle crashes were analysed. The gender distribution was 97 (93\%) males and 7 (7\%) females with ages ranging between 14 and 64 years (mean 38 years, SD 14). Of the crashes, 42 (40\%) occurred during racing and $62(60 \%)$ occurred during practice runs. Lowsider crashes represented 67 (68\%) accidents, highsider crashes accounted for $20(20 \%)$ accidents and 12 (12\%) were caused by collisions with other motorcycles (data were missing for five crashes). Crash speeds (actual speed stated by the rider $n=80$, or estimated speed $n=$
24) varied from $50 \mathrm{~km} / \mathrm{h}$ to over $180 \mathrm{~km} / \mathrm{h}$, with a mean of $92 \mathrm{~km} / \mathrm{h}$ (SD 24).

In all, 30 riders (29\%) were considered injured. Of these, 25 patients (24\%) had suffered injuries (ISS 1-14) and $5(5 \%)$ sustained major trauma (ISS > 15). Sixteen riders were referred to the hospital as trauma patients. The mean ISS for those 16 patients was 8 (range 1-21; SD 6) and the mean NISS was 10 (range 1-29; SD 8.1). Five riders $(5 \%)$ were classified as having major trauma (ISS > 15) (see Table 1). None were reported dead after 30 days. Neither the motorcycle or insurance organizations nor the public local ambulance services had knowledge of any accidents on the racetrack that were not already part of the data.

The first documented vital signs were reported between 2 and 58 min after crashes, with a mean of 15 min (SD 18). In 35 (34\%) of the accidents, vital signs were reported within $3 \mathrm{~min}$ of the crash. After $5 \mathrm{~min}, 47$ accidents (45\%) had vital signs measured, and 78 cases (75\%) were accounted for within $10 \mathrm{~min}$ of the crash. Just nine accidents (8\%) had initial vital signs measured after 60 min (see Fig. 1).

\section{Vital Signs Within the First Minutes After an Accident}

The vital signs observed within the first minutes after a crash varied considerably. The first measured heart rates for all riders and the times for those observations are displayed in Fig. 1. As shown, heart rates varied between 67 and 146 beats per minute (mean 105; SD 18). When comparing the first measured heart rates among seriously injured (marked in Fig. 2) and uninjured riders, no significant difference could be observed (Fisher's exact test; uninjured riders: $n=67 ; \mathrm{M}=104 ; \mathrm{SD}=18$; injured riders: $n=28 ; \mathrm{M}=105 ; \mathrm{SD}=18 ; 95 \% \mathrm{CI}-7$ to 9, $p=0.92$ ).

The means of vital signs among uninjured, injured and seriously injured riders were compared using the Kruskal-Wallis test (see Table 2). A significantly lower Glasgow Coma Scale (GCS) was observed when comparing both uninjured (15; SD 0) with injured riders (12; SD 4) (Kruskal-Wallis test: $\mathrm{H}(2)=19 ; p=0.00$; post hoc Bonferroni adjusted; $p \leq 0.00$ ) and uninjured (15; SD 0)

Table 1 Overview of outcomes for all riders

\begin{tabular}{ll}
\hline Outcome, $\boldsymbol{N}=\mathbf{1 0 4}$ & $\mathbf{n ( \% )}$ \\
\hline Considered injured & $30(29)$ \\
Transported from track to racetrack medical centre with ambulance* & $35(34)$ \\
Attended the racetrack medical centre by feet & $69(66)$ \\
Cleared on the track and returned to paddock or race & $79(76)$ \\
Referred to hospital as trauma patients & 16 (15) \\
Advised to seek to non-emergency medical care & $9(9)$ \\
Reported dead after 30 days & $0(0)$ \\
\hline
\end{tabular}

*In accordance with regulations, all riders who crashed had to visit the medical centre after the crash in order to get medical clearance 


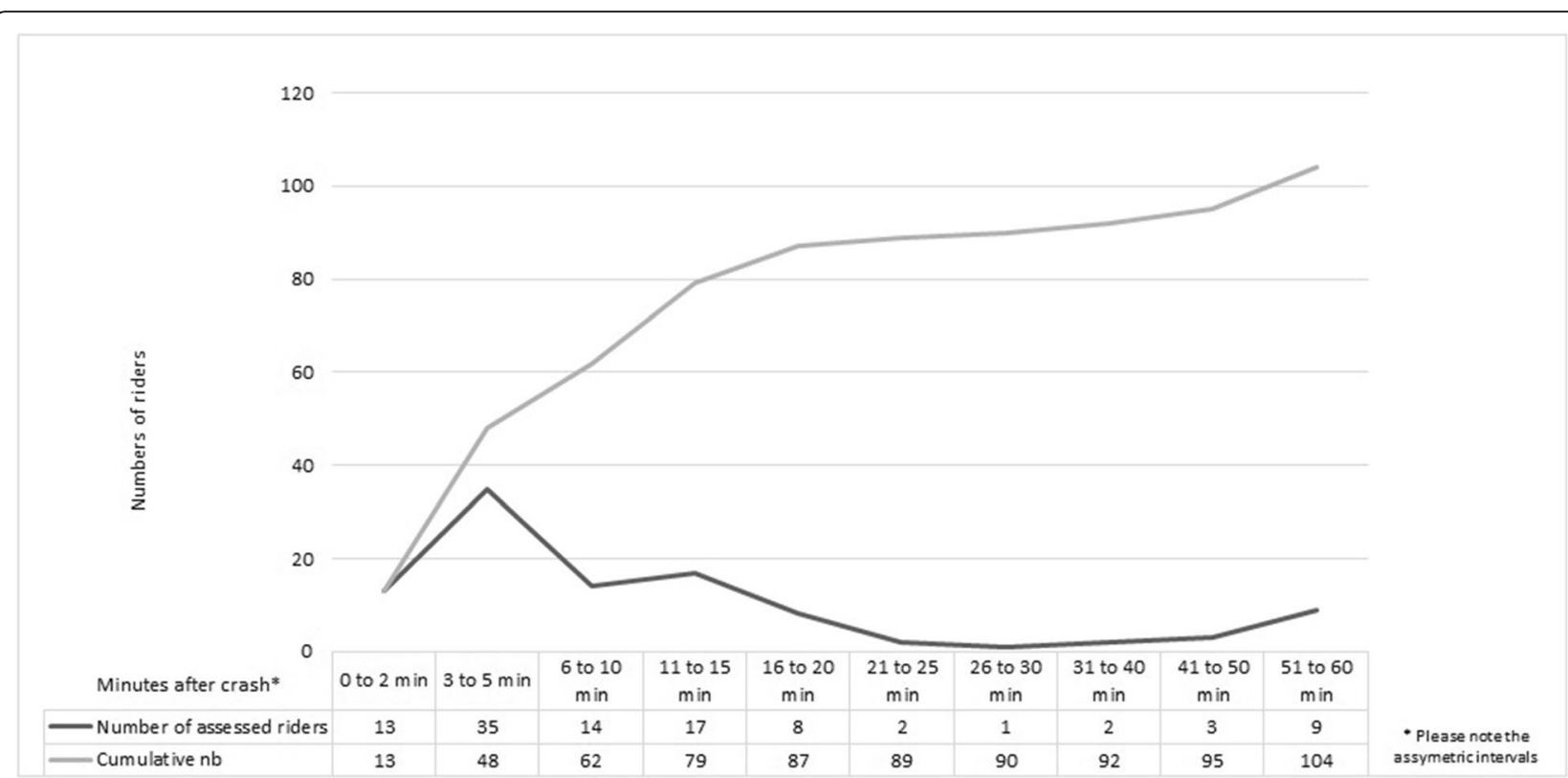

Fig. 1 Overview of time for first documented vital signs

with seriously injured riders (13; SD 2) (Kruskal-Wallis test: $\mathrm{H}(2)=19 ; p=0.00$; post hoc Bonferroni adjusted; $p \leq 0.00)$. An increased respiratory rate among seriously injured (mean 25; SD 6) compared to uninjured (mean 18; SD 2) riders was also observed (Kruskal-Wallis test: $\mathrm{H}(2)=7.6 ; p=0.22$; post hoc Bonferroni adjusted; $p=$ $0.02)$. No other vital signs were significantly different between the groups.

The development of vital signs for seriously injured patients during the first $15 \mathrm{~min}$ after an accident is shown in Table 3. Despite abnormal signs (as defined by the Swedish Trauma Protocol) in the very early phases, most vital signs were normalized when patients arrived at the hospital (see Table 3).

\section{Prehospital Clinical Signs of Injuries}

Of all 104 riders who crashed, 74 (71\%) had no signs of injury at all, while 30 (29\%) were considered as injured. The injury location was six $(20 \%)$ suffering from head injury, seven (23\%) with upper extremity injuries (i.e. arm, hand or shoulder), 10 (33\%) with lower extremity damage (i.e. leg or foot), five (17 \%) with thoracic

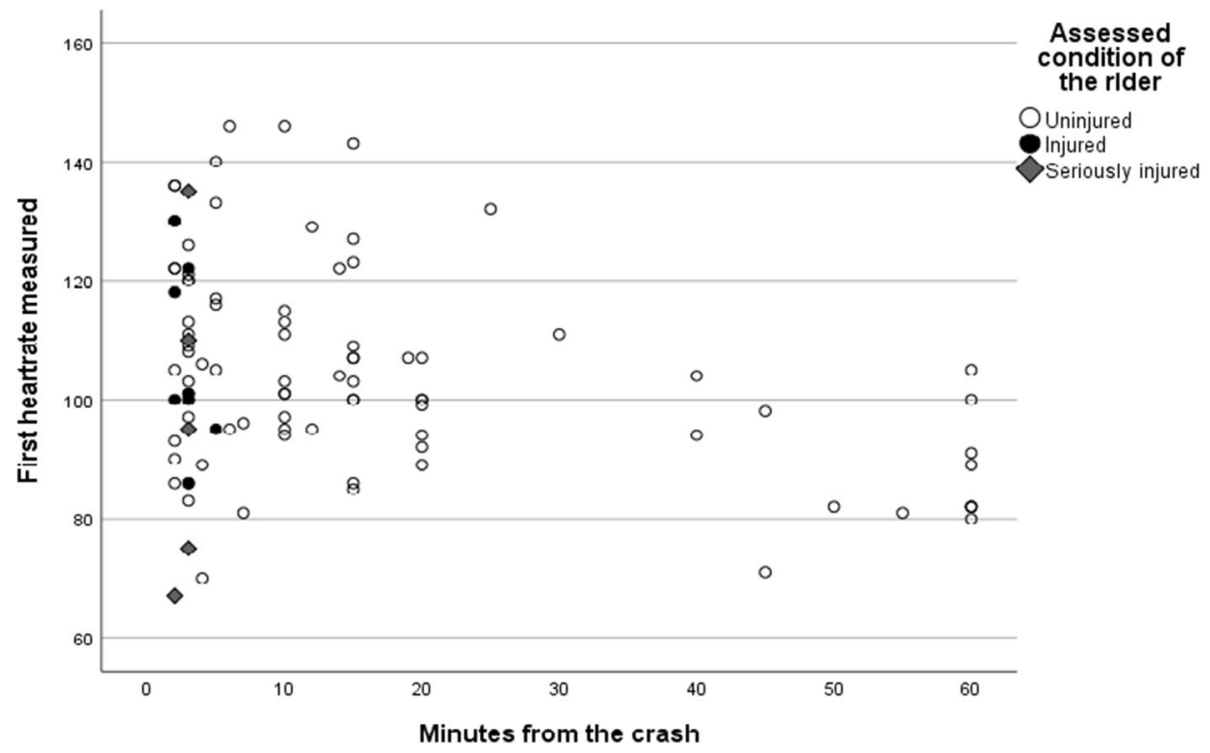

Fig. 2 First measured heart rates and times after accidents 
Table 2 Vital signs measured within the first 5 min among uninjured, injured and seriously injured riders

\begin{tabular}{|c|c|c|c|c|c|}
\hline Vital sign & $n$ & $\begin{array}{l}\text { Uninjured } \\
\text { Mean } \\
\text { (range, SD) } \\
(95 \% \mathrm{Cl})\end{array}$ & $\begin{array}{l}\text { Injured } \\
\text { Mean } \\
\text { (range, SD) } \\
(95 \% \mathrm{Cl})\end{array}$ & $\begin{array}{l}\text { Seriously injured } \\
\text { Mean } \\
\text { (range, SD) } \\
(95 \% \mathrm{Cl})\end{array}$ & $p$-value \\
\hline Heart rate & 44 & $\begin{array}{l}109 \\
(70-140, \text { SD 17) } \\
(95 \% \text { Cl 102-116) }\end{array}$ & $\begin{array}{l}107 \\
(86-130, \text { SD 16) } \\
(95 \% \text { Cl 92-102) }\end{array}$ & $\begin{array}{l}102 \\
(67-135, \text { SD 28) } \\
(95 \% \text { Cl 56-147) }\end{array}$ & 0.34 \\
\hline Respiratory rate & 40 & $\begin{array}{l}18 \\
(16-22, S D 2) \\
(95 \% \text { Cl 17-18) }\end{array}$ & $\begin{array}{l}18 \\
(16-22, S D 2) \\
(95 \% \text { Cl 16-21) }\end{array}$ & $\begin{array}{l}25 \\
(18-32, S D 6) \\
(95 \% \text { Cl 18-25) }\end{array}$ & $0.02^{* *}$ \\
\hline $\mathrm{SpO} 2$ & 46 & $\begin{array}{l}96 \\
(94-98, \text { SD 1) } \\
(95 \% \text { Cl } 95-96)\end{array}$ & $\begin{array}{l}95 \\
(92-98, \text { SD 2) } \\
(95 \% \text { Cl 93-97) }\end{array}$ & $\begin{array}{l}94 \\
(90-97, \text { SD 3) } \\
(95 \% \text { Cl } 90-99)\end{array}$ & 0.82 \\
\hline Systolic blood pressure & 43 & $\begin{array}{l}139 \\
(104-189, \text { SD 20) } \\
(95 \% \text { Cl 131-147) }\end{array}$ & $\begin{array}{l}141 \\
(105-190, \text { SD 32) } \\
(95 \% \text { Cl 112-171) }\end{array}$ & $\begin{array}{l}133 \\
(112-163, \text { SD 23) } \\
(95 \% \text { Cl } 96-170\end{array}$ & 0.08 \\
\hline GCS & 48 & $\begin{array}{l}15 \\
(15-15, \text { SD 0) } \\
(95 \% \text { Cl } 15-15)\end{array}$ & $\begin{array}{l}12 \\
(3-15, \text { SD 4) } \\
(95 \% \mathrm{Cl} 11-15)\end{array}$ & $\begin{array}{l}13 \\
(12-15, \text { SD 2) } \\
(95 \% \text { Cl 11-16) }\end{array}$ & $<0.00^{* *}$ \\
\hline
\end{tabular}

SpO2, peripheral capillary oxygen saturation; SBT, systolic blood pressure; GCS Glasgow Coma Scale

*Analysed with the Kruskal-Wallis test. ${ }^{* *}$ For significant results, post hoc tests using Bonferroni were made to compare pairs within the groups (see text)

injuries and two (6 \%) suffering from suspected spinal cord injury. When comparing prehospital and hospital assessments of injury locations, there was a $75 \%$ match for the 16 patients transferred to the hospital. Five of the patients (31\%) had additional injuries identified by the hospital trauma team as compared to the documented prehospital assessment. These included fractures or soft tissue injured on more locations than identified prehospital. None of the additional injuries found were considered as life threatening or severe on their own but added severity to the overall condition of the injured rider.

\section{Association Between Trauma Mechanisms, Vital Signs Measured Early and Injuries}

The occurrence of injuries was associated with highsider or collision crashes, a prehospital decreased level of consciousness (GCS < 15) and clinical signs of injuries; the type of crash had the greatest impact (see Table 4).

\section{Dropouts}

A few patients had incomplete vital sign documentation in any or several readings (heart rate $(n=3)$, breathing frequency $(n=2)$, systolic blood pressure $(n=3))$ and three $(n=3)$ had no documented $\mathrm{SpO} 2$ assessments but all other data. No specific dropout analysis was conducted due to the small number of participants.

\section{Discussion}

This study has shown that about $30 \%$ of all road racing crashes on the racetrack during practice and racing caused injuries, and in $5 \%$ of these, the riders were severely injured. Vital signs in the early phases after crashes varied significantly. Aside from a decreased level of consciousness, no single vital sign or kinematic component observed shortly after a crash was a strong indicator of neither injuries nor severe injuries. However,

Table 3 Overview of seriously injured riders' vital signs during the first 15 min after a crash

\begin{tabular}{|c|c|c|c|c|c|c|c|c|c|c|c|c|c|c|c|c|c|c|c|c|c|c|}
\hline \multirow[b]{2}{*}{ Patient } & \multirow[b]{2}{*}{$\begin{array}{l}\text { Speed } \\
(\mathbf{k m} / \mathbf{h})\end{array}$} & \multirow[b]{2}{*}{ Age } & \multicolumn{5}{|c|}{ Minute 1 to 5 after crash } & \multicolumn{5}{|c|}{ Minute 6 to 10 after crash } & \multicolumn{5}{|c|}{ Minute 11 to 15 after crash } & \multicolumn{5}{|c|}{ At arrival to hospital } \\
\hline & & & HR & BF & Sp02 & GCS & SBP & HR & BF & Sp02 & GCS & SBT & HR & BF & Sp02 & GCS & SBT & HR & BF & Sp02 & GCS & SBT \\
\hline $\mathbf{A}$ & 180 & 53 & 75 & n.a & 95 & 12 & n.a & 75 & 14 & 95 & 13 & n.a & 81 & 16 & 96 & 15 & n.a & 76 & 18 & 97 & 15 & 175 \\
\hline B & 75 & 32 & 95 & 32 & 95 & 15 & 112 & 88 & 30 & 97 & 15 & 108 & 88 & 26 & 96 & 15 & 110 & 90 & 25 & 98 & 15 & 115 \\
\hline C & 75 & 63 & 67 & 22 & 90 & 12 & 117 & 60 & 22 & 98 & 15 & 73 & 50 & 20 & 99 & 15 & 87 & 76 & 20 & 96 & 15 & 95 \\
\hline D & 180 & 50 & 110 & 18 & 95 & 15 & 140 & 100 & 16 & 96 & 15 & 130 & n.a & n.a & n.a & n.a & n.a & 105 & 18 & 95 & 15 & 140 \\
\hline $\mathbf{E}$ & 120 & 35 & 135 & 29 & 95 & 12 & 163 & 100 & 18 & 96 & 15 & 150 & n.a & n.a & n.a & n.a & n.a & 97 & 20 & 97 & 15 & 140 \\
\hline
\end{tabular}

$\mathrm{HR}=$ heart rate, $\mathrm{BF}=$ breathing frequency, $\mathrm{SpO} 2=$ peripheral capillary oxygen saturation, $\mathrm{CGS}=$ Glasgow Coma Scale, $\mathrm{SBT}=$ systolic blood pressure

n.a $=$ information not available. Grey marking $=$ abnormal vital sign according to the Swedish trauma team's activation protocol.

$H R$, heart rate; $B F$, breathing frequency; SpO2, peripheral capillary oxygen saturation; GCS, Glasgow Coma Scale; SBT, systolic blood pressure; .a, information not available; grey marking, abnormal vital sign according to the Swedish trauma team's activation protocol 
Table 4 Correlation between first measured vital signs within 10 min from the crash, speed, age of rider, type of crash and occurrence of injuries

\begin{tabular}{lllll}
\hline & $\boldsymbol{B}$ & $\mathbf{9 5 \%} \mathbf{C l}$ & $\boldsymbol{t}$ & Sig. \\
\hline Speed $\geq \mathbf{9 0} \mathbf{~ k m} / \mathbf{h}$ & 0.02 & $(-0.06 ; 0.06)$ & 0.56 & 0.59 \\
Highsider or collision & 0.92 & $(0.00 ; 0.19)$ & 1.94 & 0.05 \\
Age $\geq \mathbf{4 0}$ years & -0.12 & $(-0.11 ; 0.85)$ & -0.25 & 0.81 \\
$\mathbf{G C S}<\mathbf{1 5}$ & 0.27 & $(0.74 ; 0.46)$ & 2.75 & 0.00 \\
Respiratory rate & 0.15 & $(-0.14 ; 0.44)$ & 1.04 & 0.30 \\
$<\mathbf{1 0}$ or $>\mathbf{2 9}$ & & & & \\
SpO2 $<\mathbf{9 5 \%}$ & 0.03 & $(-0.18 ; 0.25)$ & 0.31 & 0.76 \\
Heart rate & -0.10 & $(-0.20 ;-0.00)$ & -2.24 & 0.08 \\
$<\mathbf{5 0}$ or $>\mathbf{1 2 0}$ & & & & \\
SBT $<\mathbf{9 0}$ & 0.13 & $(-0.03 ; 0.29)$ & 1.56 & 0.17 \\
Clinical signs of injury & 0.68 & $(0.57 ; 0.78)$ & 12.6 & 0.00 \\
\hline
\end{tabular}

Model summary: $p=0.00, R^{2}=0.93$. Outcome variable: injuries

$B$, unstandardized coefficient beta; $\mathrm{Cl}$, confidence interval (lower bound; upper bound); Sig., $p$ value; cursive number, significant $p$ value. GCS, Glasgow Coma

Scale; SBT, systolic blood pressure; SpO2, peripheral capillary oxygen saturation

weak links may exist between highsider or collision crashes and the occurrence of injuries.

A decreased level of consciousness was more common among injured or severely injured riders compared with uninjured riders and was associated with the occurrence of injuries in the regression analysis. The results showed that the injured group had a lower mean GCS that the serious injured group. Also, it should be noted that two riders had normal GCS both at the racetrack and when arriving to hospital but were still severely injured. None of these two was diagnosed with traumatic brain injury. Since the number of injured among these subgroups were small, no conclusions can be drawn from these differences. The GCS has been found to show strong agreement when prehospital assessments were compared with emergency department assessments [11]. However, the patients in this study who presented a prehospital lowered level of consciousness showed normal levels when arriving at the hospital. It should be noted that in this study, medical professionals reached the patients within minutes of their crashes and could make professional assessments of consciousness levels. Normally, when ambulances arrive awhile after accidents, assessments of early decreased consciousness must rely on information gathered from bystanders or patients themselves. Such information may be less reliable or unavailable, depending on the circumstances. The current findings might be due to the presence of medical professionals, or from the very early clinical assessment made. The use of vital signs in prehospital assessments of trauma patients has been questioned $[12,13]$. Within a few minutes of highspeed motorcycle crashes, vital sign variation was considerable. Some uninjured riders had heart rates over 120 during the first minutes without being seriously injured, while others had normal vital signs but were severely injured. One reason for this finding may be the physical demands of road racing, which make it difficult to distinguish between pathological high heart or respiratory rates shortly after a crash, or the physical fitness of the riders. Vital signs among the severely injured riders showed some abnormal values but also considerable variation during the first $15 \mathrm{~min}$. Since clinical decision-making in the prehospital context is a complex process [14] where each patient must be examined thoroughly to avoid any misinterpretation of conditions [6, 14] repeated observations and trends over time may be more reliable and of greater clinical value than single measurements for predicting injury severity in a very early phase. This study could not confirm if vital signs measured later were more reliable as indicators of injury severity than vital signs measured early. Neither the observed mismatch of injury location nor the clinical effects of this mismatch could be concluded from the available data. However, the results indicate that timing and context must also be considered in such assessments. This is important to consider for medical teams on standby in high-risk sports.

According to the Swedish trauma team's activation protocol, crash speeds over $35 \mathrm{~km} / \mathrm{h}$ require trauma team activation for motorcycle accidents [15]. In this study, crashes occurred at actual or estimated speeds from 50 to $180 \mathrm{~km} / \mathrm{h}$, but no association was found between speed and the occurrence of injuries. However, $23 \%$ of the speed data in this study relied on estimated speeds. The estimations were based on professional or semi-professional riders and might therefore not be representative for nonprofessional riders. Despite these limitations, the findings indicate that crash speeds may not be a good indicator to previse the severity of motorcycle injury on racetracks. By contrast, the results suggest that highsider and collision crashes could be a better indicator in these settings; this was also observed by Bedolla et al. [6]. An adjustment of general trauma triage protocols could therefore be considered for specific contexts such as racetrack accidents, in order to provide guidance on prehospital trauma triage.

There are significant differences between racetracks and public roads. At racetracks, bikers are often experienced and trained, having safety zones and mandatory personal protection gear that gradually dissipates energy in case of a crash. Motorbikes on public roads must share the road with other types of vehicles, some travelling in the opposite direction. Moreover, public bikers have the choice of going without protective gear. Another difference is that professional or semi-professional riders most often are elite athletics. Therefore, generalizing these findings to public motorcycling should be done with caution. The low rate of serious injuries despite 
extremely high speeds could indicate that the protective measures on racetracks are effective in reducing the risk of severe injuries compared with public roads. Regardless, crashes at racetracks offer a special opportunity to collect early crash data and may provide valuable insights into motorcycle crashes and prehospital clinical assessments that could benefit the larger, noncompetitive motorcycle riding population $[6,16]$. The study's setting, where medical professionals were able to respond within minutes of an accident, offers knowledge that cannot be obtained easily in ordinary prehospital trauma care or registers. Similar settings are available in other sports, such as alpine skiing. Using data from these settings offers a unique possibility to further understand the initial timeframe after accidents. Therefore, this study has value despite the limited number of crashes included. The results of this study raise several questions concerning prehospital clinical assessment of trauma patients. Further research should be undertaken to investigate the development over time of vital signs in trauma patients and further discuss their value for prehospital assessment. Additionally, the value of kinematic data in prehospital trauma triage protocols for different settings and activities needs to be further explored.

\section{Limitations}

The study has several limitations. First, the number of severely injured patients in this study sample was very small, reducing the possibility of generalizing the results. However, this is also a finding itself, that fewer serious injuries than expected occurred at the racetrack. The available data only cover accidents that occurred on a racetrack when medical professionals were on standby. Despite regulations, it cannot be excluded that any road racing activities were conducted without the medical team on standby, and therefore not covered in this study. In addition, there may have been late complications or injuries requiring medical care that were not covered in this study-for example, if the patient had sought medical care in another country (e.g. their home country). However, the screening of insurance companies, motorcycle organizations and public ambulance services showed that they had no information on fatalities due to accidents on racetracks during the period studied. The SweTrau register reports both ISS and NISS for each incident. As the ISS and NISS have been reported to have a similar ability of predicting mortality in trauma patients [17], this study used the ISS only to define severe injuries in the analysis.

\section{Conclusions}

Vital signs measured within a few minutes of riders being involved in motorcycle crashes on a racetrack varied considerably. Except for a decreased level of consciousness, the clinical value for prehospital assessment of vital or clinical signs measured early might, therefore, be limited. The type of crash seemed to be a better indicator of severe injuries than estimated speed at crash, and it could be considered to adapt general trauma triage protocols to the racetrack settings. Using the context of a study setting with medical professionals at the victim's side within a few minutes after an accident offers unique possibilities to increase our understanding of clinical assessment in the very early state after an accident. Therefore, such study settings may benefit both sports medicine contexts and general trauma care.

\section{Abbreviations \\ BF: Breathing frequency; Cl: Confidence interval; GCS: Glasgow Coma Scale; HR: Heart rate; ISS: Injury severity score; NISS: New injury severity score; SpO2: Peripheral capillary oxygen saturation; SD: Standard deviations; SweTrau: Swedish Trauma Registry; SBT: Systolic blood pressure}

\section{Acknowledgements}

Not applicable

\section{Authors' Contributions}

$\mathrm{KH}$ and $\mathrm{J}$ planned the study, and $\mathrm{KH}$ obtained ethical permissions. Data were collected by JL and LE (at the racetrack) and KH (data collected from SweTrau). Data management and statistical analysis were conducted by $\mathrm{KH}$ with the assistance of an external statistician. PÖ and $\mathrm{KH}$ contributed to the final protocol and data interpretation. $\mathrm{KH}$ drafted the manuscript. All authors contributed substantially and approved the final version as submitted.

\section{Funding}

Open Access funding provided by Örebro University.

\section{Availability of Data and Materials}

The datasets generated during and analysed during the current study are available from the corresponding author on reasonable request.

\section{Declarations}

Ethics Approval and Consent to Participate

Ethical approval was obtained from the Swedish Ethical Review Authority (dnr 2019-05803).

\section{Consent for Publication}

Not required

\section{Competing Interests}

One author, $J$, is the owner of the private medical services company contracted as a medical team at the racetrack studied. The other authors, Karin Hugelius, Linda Ekh and Per Örtenwall, declare that they have no competing interests.

\section{Author details}

${ }^{1}$ Faculty of Medicine and Health, Örebro University, 70182 Örebro, SE, Sweden. ${ }^{2}$ Region Örebro County, Karlskoga Hospital, 69144 Karlskoga, Sweden. ${ }^{3}$ TM: s ambulance, 65226 Karlstad, Sweden. ${ }^{4}$ Department of Clinical Physiology, Region Jämtland Härjedalen, 83131 Östersund, Sweden.

${ }^{5}$ Sahlgrenska Academy, Gothenburg University, 40530 Gothenburg, Sweden.

Received: 19 February 2021 Accepted: 28 July 2021

Published online: 21 August 2021

\section{References}

1. Svenska motorsportförundet (eng: Swedish Association of Motorsport). Special regulations for road racing. Norrkoping; 2021. 
2. D'Artibale E, Rohan M, Cronin JB. Trend analysis of 20 years of FIM road racing grand prix motorcycle world championship. Int J Sports Physiol Perform. 2018;13(6):795-801. https://doi.org/10.1123/ijspp.2017-0664.

3. WHO. Global status report on road safety 2018. Geneva: WHO; 2018

4. Swedish Transport Agency. National Statistics on traffic accidents 2019, Swedish Stockholm. 2019.

5. (SKL) Swedish Association of Local Authorities and Regions. Statistical yearly report of ambulance services in Sweden. Stockholm: 2019.

6. van Rein EAJ, Houwert RM, Gunning AC, Lichtveld RA, Leenen LPH, van Heijl M. Accuracy of prehospital triage protocols in selecting severely injured patients: a systematic review. J Trauma Acute Care Surg. 2017;83(2):328-39. https://doi.org/10.1097/ta.0000000000001516.

7. Bedolla J, Santelli J, Sabra J, Cabanas JG, Ziebell C, Olvey S. Elite motorcycle racing: crash types and injury patterns in the MotoGP class. The American journal of emergency medicine. 2016;34(9):1872-5. https://doi.org/10.1016/ j.ajem.2016.07.005

8. Keil $H$, Reimers $D$, Lingner $M$, et al. Injury patterns in professional motorbike racing: a retrospective analysis of 728 falls. J Sports Med Phys Fitness. 2019; 59(5):817-21. https://doi.org/10.23736/s0022-4707.18.09056-4.

9. Stevenson M, Segui-Gomez M, Lescohier I, di Scala C, McDonald-Smith G. An overview of the injury severity score and the new injury severity score. Inj Prev. 2001;7(1):10-3. https://doi.org/10.1136/ip.7.1.10.

10. Bland M. An Introduction to Medical Statistics. 3rd ed. Oxford, Great Britain: Oxford University Press; 2000.

11. Trust MD, Schellenberg M, Biswas S, Inaba K, Cheng V, Warriner Z, et al. Prehospital vital signs accurately predict initial emergency department vital signs. Prehosp Disaster Med. 2020;35(3):1-6. https://doi.org/10.1017/s104 $9023 \times 2000028 x$

12. Liu NT, Holcomb JB, Wade CE, Salinas J. Inefficacy of standard vital signs for predicting mortality and the need for prehospital life-saving interventions in blunt trauma patients transported via helicopter: a repeated call for new measures. J Trauma Acute Care Surg. 2017;83(1 Suppl 1):s98-s103. https:// doi.org/10.1097/ta.0000000000001482.

13. Najafi Z, Abbaszadeh A, Zakeri $H$, et al. Determination of mis-triage in trauma patients: a systematic review. Eur J Trauma Emerg Surg. Published Online First: 25 February 2019. https://doi.org/10.1007/s00068-019-01097-2

14. Andersson U, Maurin Soderholm H, Wireklint Sundstrom B, et al. Clinical reasoning in the emergency medical services: an integrative review. Scand J Trauma Resusc Emerg Med. 2019;27(1):76. Published Online First: 21 August 2019. https://doi.org/10.1007/s00068-019-01097-2.

15. Linder F, Holmberg $L$, Bjorck $M$, et al. A prospective stepped wedge cohort evaluation of the new national trauma team activation criteria in Sweden - the TRAUMALERT study. Scand I Trauma Resusc Emerg Med. 2019;27(1):52. Published Online First: 2 May 2019. https://doi.org/10.1186/s13 049-019-0619-1.

16. Tomida $\mathrm{Y}$, Hirata $\mathrm{H}$, Fukuda $\mathrm{A}$, et al. Injuries in elite motorcycle racing in Japan. Br J Sports Med. 2005;39(8):508-11. https://doi.org/10.1136/bjsm.2 004.013722 [published Online First: 28 July 2005].

17. Deng $Q$, Tang $B$, Xue $C$, et al. Comparison of the ability to predict mortality between the Injury Severity Score and the New Injury Severity Score: a meta-analysis. Int J Environ Res Public Health. 13(2016, 8):825. https://doi. org/10.3390/ijerph13080825 [published Online First: 19 August 2016].

\section{Publisher's Note}

Springer Nature remains neutral with regard to jurisdictional claims in published maps and institutional affiliations.

\section{Submit your manuscript to a SpringerOpen ${ }^{\circ}$ journal and benefit from:}

- Convenient online submission

- Rigorous peer review

- Open access: articles freely available online

- High visibility within the field

- Retaining the copyright to your article

Submit your next manuscript at $\boldsymbol{\nabla}$ springeropen.com 